\title{
Análisis de las relaciones lodo cemento como material de construcción no estructural
}

\author{
Natalia Fuentes-Molina ${ }^{\star}$, Darlys N. Cujia-Urrutia ${ }^{2}$ y Carlos A. Robles-Julio \\ (1) Facultad de Ingenierías, Grupo de Investigación Ciencia + Ambiente, Universidad de La Guajira, Km 5, vía a Maicao \\ Riohacha. La Guajira, Colombia. (correo-e: nnfuentes@uniguajira.edu.co) \\ (2) Facultad de Ingenierías, Grupo de Investigación Ciencia + Ambiente, Universidad de La Guajira, Km 5, vía a Maicao \\ Riohacha. La Guajira, Colombia. (correo-e: dncujia@uniguajira.edu.co)
}

* Autor a quien debe ser dirigida la correspondencia

Recibido Abr. 28, 2021; Aceptado Jun. 23, 2021; Versión final Ago. 14, 2021, Publicado Dic. 2021

\begin{abstract}
Resumen
La presente investigación evaluó las condiciones óptimas de uso de lodo aluminoso, de un sistema de tratamiento de agua potable, como sustituto parcial del cemento para su uso en la fabricación de materiales de construcción no estructurales. Se prepararon mezclas estándar de lodo aluminoso (5, 10, 15 y $20 \%)$ con concreto. A los 7, 28 y 42 días de curado, se realizaron análisis de granulometría, fluorescencia de rayos $\mathrm{X}$, materia orgánica, humedad, resistencia a compresión y absorción. Los resultados registraron diferencias significativas con variaciones de resistencia a la compresión entre 2.60 a $7.70 \mathrm{Mpa}$. Las mezclas del 15 y $20 \%$ fueron las únicas que cumplieron con los límites establecidos para materiales de construcción no estructural de la norma ASTM-C129. Se concluye que la introducción de lodo aluminoso (15 y 20\%) como sustituto del cemento no deteriora la resistencia mecánica del material.
\end{abstract}

\section{Chemical analysis of the cement-sludge interaction as a non- structural construction material}

\begin{abstract}
The present research study examined the optimal conditions for using aluminous sludge, from a drinking water treatment system, as a partial substitute for cement to manufacture non-structural construction materials. Standard mixtures consisted of aluminous sludge (5, 10, 15 and 20\%) and cement. At 7, 28, and 42 days, mixtures were examined for granulometry, X-ray fluorescence, organic matter content, humidity, compressive resistance, and absorption. The results showed significant differences with variations in compressive strength between 2.60 and $7.70 \mathrm{MPa}$. The mixtures with 15 and $20 \%$ aluminous sludge were the only ones that met the limits established for non-structural building materials by the ASTM-C129 regulation. In conclusion, introducing aluminous sludge ( 15 and $20 \%$ ) as a substitute for cement did not deteriorate material mechanical resistance.
\end{abstract}

Keywords: aluminous sludge; cement; compressive strength; cylinders 


\section{INTRODUCCIÓN}

En las últimas décadas, la alta demanda de materiales de construcción a un ritmo y escala sin precedentes con tendencias a continuar (Bohórquez et al., 2020; Medeiros et al., 2018), es una de las principales motivaciones para mejorar en productividad, competitividad y sostenibilidad ambiental en el sector de la construcción con nuevos paradigmas de economía circular (De Carvalho et al., 2019; Durante et al., 2018) como respuestas a los grandes desafíos globales de escasez de recursos ante escenarios de sobreexplotación, haciendo énfasis en la gestión eficiente de los materiales, orientada a reajustar los modelos de desarrollo, en forma útil y oportuna.

Estudios globales han demostrado que la industria de la construcción tiene implícito el consumo de grandes cantidades de recursos materiales y energéticos, demandando cerca de un $40 \%$ de energía primaria, y un $60 \%$ de agregados pétreos, que causan un $33 \%$ de emisiones de dióxido de carbono $\mathrm{CO}_{2}$ (León y Guillén, 2020; Liu et al., 2018), convirtiendo esta actividad en una de las más contaminantes (Gessa y Sancha 2016; Zhang et al., 2014). El panorama presentado muestra el gran potencial que tiene el sector para buscar soluciones alternativas innovadoras viables que minimicen los impactos generados como lo plantean las revisiones de Medeiros et al., (2018) y De Carvalho et al., (2019).

En concordancia con lo anterior; la producción de cemento es responsable de aproximadamente un $8 \%$ de las emisiones globales de dióxido de carbono $\mathrm{CO}_{2}$, siendo una de las mayores fuentes de gases de efecto invernadero (León y Guillén, 2020; Chen et al., 2016), con emisiones directas hasta un 60\% que en gran parte provienen de las reacciones químicas del proceso de clinkerización, y el $40 \%$ restante proviene de emisiones indirectas de la combustión, extracción de materias primas y otras (Gessa y Sancha 2016); Aún así, se presenta un incremento de la producción del cemento los últimos años por encima de los 4,65 billones ton/ anuales (Gessa y Sancha 2016; Chen et al., 2016) con un consumo per-cápita de $616 \mathrm{~kg} / \mathrm{hab}$ (León y Guillén, 2020) que refleja la urgencia de una gestión sostenible más respetuosa con el medio ambiente, en los cuales el reciclaje y reutilización de residuos como sustitutos parciales están por encima de la eliminación y se ha convertido en un tema de interés ambiental, por la necesidad de crear alternativas de aplicaciones en el uso de materiales secundarios.

En este contexto, sumando a lo anterior, se encuentran las enormes cantidades de lodos aluminosos, considerados residuos peligrosos, que se han convertido en una problemática insostenible que demanda un tratamiento y manejo alternativo viable, dada sus características físicas, químicas y microbiológicas (Odimegwu et al., 2018); existen varios escenarios para la recuperación, reutilización y el reciclaje de los lodos residuales con fines prácticos, entre los que se resaltan: $i$. la recuperación energética que tiene un desafío técnico en los procesos, reacciones de recuperación y limitaciones tecnológicas para la digestión anaeróbica, combustión, pirolisis y/o gasificación (Maslon et al., 2020; Vatachi, 2019); ii. la recuperación de suelos en los sectores agrícolas y forestales (Dassanayake. et al., 2015; Smol et al., 2015), con limitaciones por la toxicidad del aluminio (Kluczka et al., 2017); iii. la recuperación del aluminio residual en pH bajos con rendimientos de recuperación del 65 al 75\% (Gastaldini et al., 2015; Franco et al., 2015); iv. como reemplazo parcial de áridos y agregados en la producción del concreto aumentando la vida útil de las canteras (Gastaldini et al., 2015; Fuentes et al., 2019) y v. su uso como sustituto del cemento para reducir los impactos de la industria del cemento y compensar su alta demanda (Ibrahim et al., 2020; Hagemann et al., 2019), pasando a una posible disposición segura, con menores impactos ambientales y reducción de costos de disposición. A pesar de existir diferentes investigaciones que acrediten el aprovechamiento de este residuo, es necesario ampliar el conocimiento de su viabilidad en los diferentes sectores.

El interés de centrar la investigación en el sector de la construcción es justificado por las propiedades puzolánicas de los lodos residuales, que se han encontrado a través de varios estudios (Hagemann et al., 2019; Gastaldini et al., 2015), que muestran su uso como aditivo y/o agregado mineral; la literatura describe que es posible conseguir la misma resistencia observada en el concreto de referencia, reduciendo el consumo de cemento en concretos que utilizan lodos residuales. La inclusión de los lodos aluminosos en morteros como material de cemento suplementario de la masa de cemento aumenta la porosidad influyendo en todas las propiedades físicas y mecánicas, manteniendo inalterada stado general de los morteros relacionado con el comportamiento en estado fresco y endurecido (Gastaldini et al., 2015).

Su afinidad con el cemento y la capacidad de intercambio iónico hace posible su aprovechamiento mediante procesos de solidificación- estabilización (Vouk et al., 2018), es así como los lodos aluminosos se han incorporado en la elaboración de materiales de construcción, el uso de este material residual tiene resultados positivos en las propiedades físicas y mecánicas de los materiales, tales como peso ligero, porosidad y resistencia a la compresión, demostrado por diversas investigaciones (Hagemann et al., 2019; De Carvalho et al., 2019). Por consiguiente en el presente estudio se evaluó las condiciones óptimas de los lodos aluminosos generados en las plantas de tratamiento de agua potable, como agregado en la elaboración de materiales cementantes, para su uso como material de construcción no estructural. 


\section{MATERIALES Y MÉTODOS}

En el desarrollo de la investigación se siguió la metodología que se presenta en la Figura 1. Inicialmente se realizó el proceso de tamizado para la caracterización química mediante fluorescencia de rayos X (FRX), el análisis granulométrico y termo-gravimétrico, la actividad puzolánica establecida en la norma ASTM C618 y el efecto de la incorporación de los lodos residuales en las propiedades del concreto en estado endurecido.

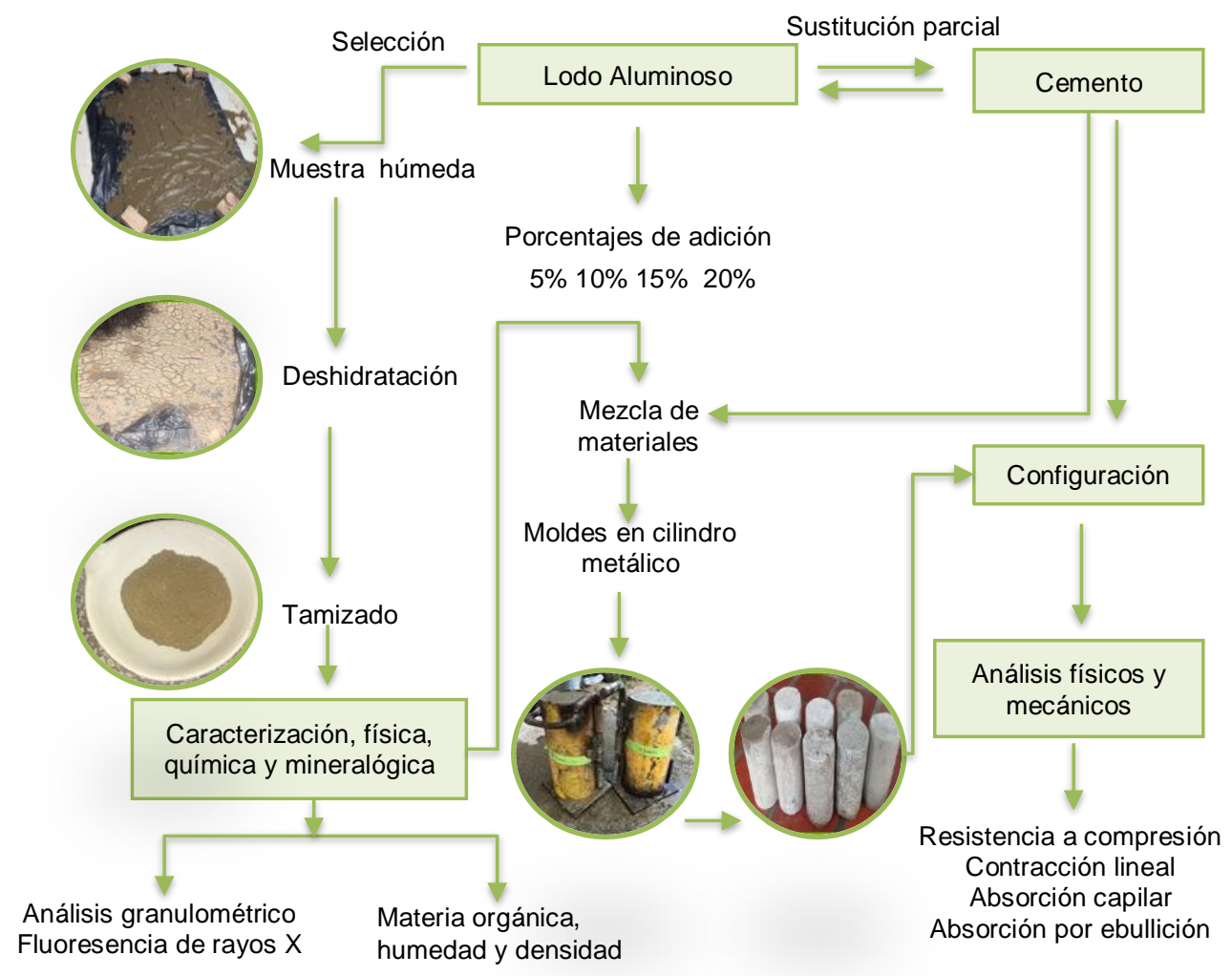

Fig. 1: Fases de análisis experimental de las relaciones lodo-cemento como material de construcción no estructural.

La evaluación de la actividad puzolánica se determinó sumando los componentes químicos $\mathrm{SiO}_{2}+\mathrm{Al}_{2} \mathrm{O}_{3}$ $+\mathrm{Fe}_{2} \mathrm{O}_{3}$, el índice de la actividad puzolánica (IAP) que es la relación entre la resistencia a la compresión con remplazo parcial del $20 \%$ de los lodos residuales en peso del cemento con los de referencia $(100 \%$ cemento portland). El concreto se elaboró con diferentes porcentajes de sustitución del cemento portland 5, 15,10 y 20 $\%$ en peso de lodos aluminosos, mediante la evaluación de cilindros de 4 × 8 in acorde a lo especificado en la norma ASTM-192, utilizando las relaciones de agua, cemento, agregado fino y lodo aluminosos presentadas en la Tabla 1 logrando una consistencia adecuada en todas las mezclas.

En razón de determinar la mejor respuesta de las mezclas frente a la prueba de resistencia a la compresión, con diferentes porcentajes de adición, se realizaron los análisis para los 7, 28 y 42 días de curado, con el propósito de conocer las reacciones puzolánicas; también se determinaron la porosidad y absorción de agua, bajo el procedimiento descrito en la norma ASTM C642 y las demás propiedades físicas teniendo en cuenta las normas ASTM-C129 y ASTM-C90. Para verificar el cumplimiento establecido en la normativa, los concretos elaborado fueron evaluados con la norma ASTM-C129 para el uso no estructurales y la norma ASTM-C90 para el uso estructural que establecen como resistencia a la compresión mínima 6.08 y $8.00 \mathrm{MPa}$ respectivamente.

Tabla 1: Relación de masas utilizadas en la elaboración del concreto según los porcentajes de adición.

\begin{tabular}{lcrrrr}
\hline \multirow{2}{*}{ Materias primas } & \multicolumn{5}{c}{ Masa utilizada $(\mathrm{kg})$} \\
\cline { 2 - 5 } & Referencias & $5 \%$ & $10 \%$ & $15 \%$ & \multicolumn{1}{c}{$20 \%$} \\
\hline Agua $(\mathrm{L})$ & 1.62 & 1.62 & 1.62 & 1.62 & 1.62 \\
Cemento $\left(\mathrm{kg} / \mathrm{m}^{3}\right)$ & 5.00 & 0.25 & 0.50 & 0.75 & 1.00 \\
Relación A / C & 0.32 & 0.32 & 0.32 & 0.32 & 0.32 \\
Agregado fino $\left(\mathrm{kg} / \mathrm{m}^{3}\right)$ & 57.50 & 57.50 & 57.50 & 57.50 & 57.50 \\
Lodo aluminoso $\left(\mathrm{kg} / \mathrm{m}^{3}\right)$ & 0.00 & 4.75 & 4.50 & 4.25 & 4.00 \\
\hline
\end{tabular}




\section{RESULTADOS}

El análisis granulométrico de los lodos aluminosos se clasifica como A2 de acuerdo con American Association of State Highway and Transportation Officials (AASHTO), y según el sistema unificado de clasificacion de suelos (USCS) de arena bien gradada SW, es notable que el porcentaje más alto se encuentra en diámetros menores a $0.84 \mathrm{~mm}$ (malla No. 20), con un porcentaje de lodo aluminoso y arena de 83.33 y $77.28 \%$ respectivamente, y los porcentajes menores a $0.075 \mathrm{~mm}$ (malla No. 200), fueron 3.47 y $4.80 \%$ de lodo aluminoso y arena respectivamente; como se evidencia en la figura 2. el lodo aluminoso está compuesto granulométricamente de materiales finos, adecuados para su uso como material de construcción.

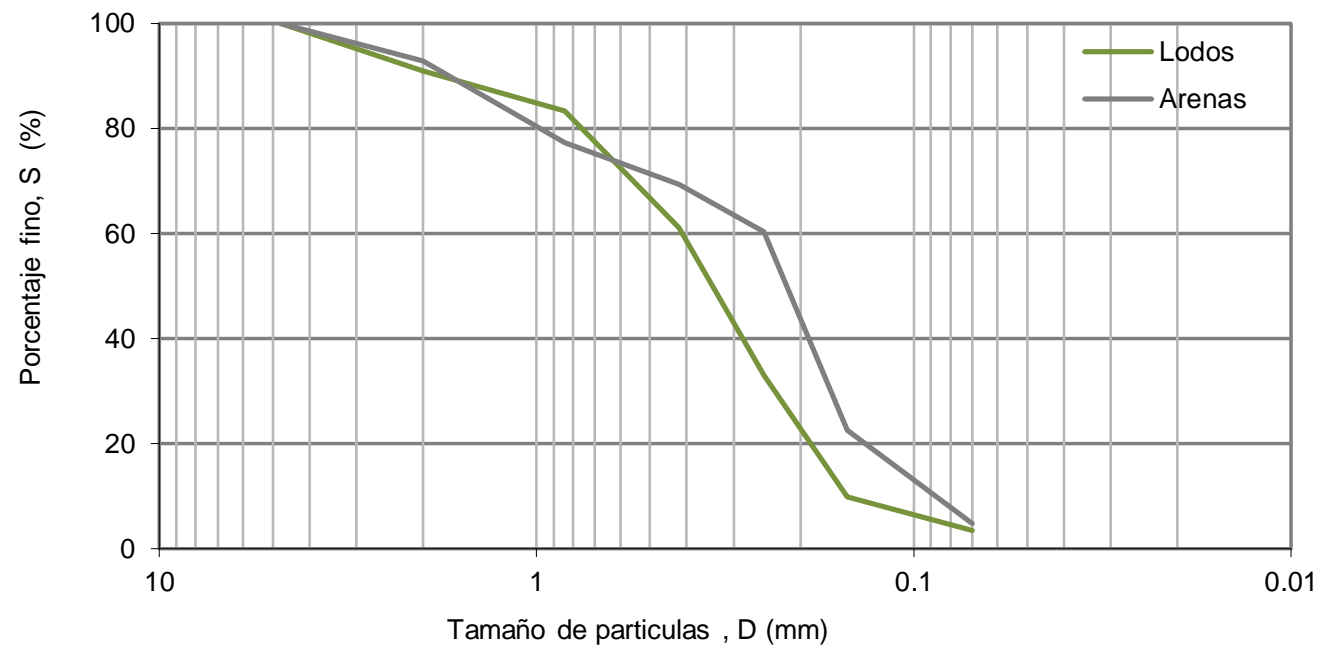

Fig. 2: Curva de distribución del tamaño de partículas

El contenido de humedad en el cemento (3.14\%) es similar al lodo aluminoso (2.80\%), y difieren en el contenido de materia orgánica en un $2.50 \%$, por otro lado, las densidades se correlacionan con la granulometría; proporcionando evidencia de que se presentan mayores densidades en el cemento (2.9 mg/L), comparado con el lodo aluminoso $(2.37 \mathrm{mg} / \mathrm{L})$ presentado en la Tabla 2 permitiendo estimar el grado de compactación y la porosidad del mismo, donde el aumento de este último con la adición de lodo aluminoso fue evidente, esto se puede atribuir a las características del lodo aluminoso y la heterogeneidad de las dos estructuras que componen las mezclas.

Tabla 2: Contenido de humedad, materia orgánica y densidad de las materias primas cemento y lodo.

\begin{tabular}{lcc}
\hline Características & Cemento & Lodo \\
\hline Contenido de humedad $(\%)$ & 3.14 & 2.80 \\
Materia orgánica $(\%)$ & -- & 2.50 \\
Densidad $(\mathrm{g} / \mathrm{mL})$ & 2.9 & 2.37 \\
\hline
\end{tabular}

Los lodos aluminosos utilizados provienen de un sistema de tratamiento de agua potable, las características físicas y químicas varían en el tiempo, en función de la calidad del afluente y los regímenes de coagulación del sistema; dado lo anterior, la presente investigación realizó el análisis químico por fluorescencia de rayos $\mathrm{X}$ de estos lodos, evidenciando las similitud con el cemento; se compone principalmente de (21.00 y 55.60 $\%)$, óxido de silicio (6.50 y $24.62 \%$ ) alúmina, (2.50 y $10.39 \%$ ) óxido de hierro y otros componentes menores como se observa en la Tabla 3. De lo anterior se puede resaltar que los compuestos mayoritarios se encuentran distribuidos en porcentajes similares, lo que beneficia el aprovechamiento del lodo por su afinidad con el cemento; facilitando la mezcla de los mismos en la elaboración del concreto.

Tabla 3: Composición química del cemento y lodos aluminosos.

\begin{tabular}{lcccccccccccccccccc}
\hline Componente & $\mathrm{SiO}_{2}$ & $\mathrm{Al}_{2} \mathrm{O}_{3}$ & $\mathrm{Fe}_{2} \mathrm{O}_{3}$ & $\mathrm{CaO}$ & $\mathrm{MgO}$ & $\mathrm{K}_{2} \mathrm{O}$ & $\mathrm{Na}_{2} \mathrm{O}$ & $\mathrm{TiO}_{2}$ & $\mathrm{P}_{2} \mathrm{O}_{5}$ & $\mathrm{SO}_{3}$ & $\mathrm{MnO}_{2}$ & $\mathrm{Ba}$ & $\mathrm{Ce}$ & $\mathrm{Cl}$ & $\mathrm{V}$ & $\mathrm{Zn}$ & $\mathrm{Sr}$ & $\mathrm{Zr}$ \\
\hline $\begin{array}{l}\text { Cemento \% } \\
\text { en peso }\end{array}$ & 21 & 6.5 & 2.5 & 62.7 & 2 & 0.8 & 0.8 & 0.25 & 0.1 & 1.5 & 1.6 & 0,02 & 0 & 0.04 & 0 & 0.02 & 0.1 & 0 \\
$\begin{array}{l}\text { Lodo \% en } \\
\text { peso }\end{array}$ & 55,60 & 24,62 & 10,39 & 2,08 & 2,05 & 2,00 & 1,22 & 0,86 & 0,34 & 0.28 & 0.24 & 0.1 & 0.05 & 0.03 & 0.03 & 0.03 & 0,02 & 0,02 \\
\hline
\end{tabular}


Tabla 4: Propiedades químicas y físicas de los lodos aluminosos

\begin{tabular}{|c|c|c|}
\hline Requerimientos & Norma ASTM C618 & Lodos \\
\hline \multicolumn{3}{|l|}{ Requerimiento Químico } \\
\hline $\mathrm{SiO}_{2}+\mathrm{Al}_{2} \mathrm{O}_{3}+\mathrm{Fe}_{2} \mathrm{O}_{3}$ & $\geq 70$ & 90,61 \\
\hline $\mathrm{SO}_{3}$ & $\leq 4$ & 0,28 \\
\hline Contenido de humedad (\%) & $\leq 3$ & 2,80 \\
\hline Pérdida de Ignición (\%) & $\leq 10$ & 8,3 \\
\hline Requerimiento Físico & & \\
\hline Índice de actividad puzolánico IAP & $\geq 75$ & 79,27 \\
\hline
\end{tabular}

En la actividad punzolánico se evidencia que la sumatoria de los compuestos $\mathrm{SiO}_{2}, \mathrm{Al}_{2} \mathrm{O}_{3}$ y $\mathrm{Fe}_{2} \mathrm{O}_{3}$ de los lodos residuales supera el $70 \%$ que se establece en la norma ASTM C618 para punzolanas naturales, es necesario seguir avanzando en el conocimiento de la estructura y morfología de estos. Por otra parte la pérdida de ignición de los lodos residuales se encuentran dentro de los límites establecidos en la norma ASTM C618 para punzolanas naturales menores al 10\% que incluyen el cloro elemental y otros elementos volátiles que presentan los lodos en su composición química, analizados por medio del análisis térmico diferencial.

El contenido de materia orgánica en el concreto mantiene una relación directamente proporcional al porcentaje de adición de lodo aluminoso, esto posiblemente se deba a las diferencias de tamaño en las partículas, generando un aumento en la porosidad que contribuye a la formación de cavidades. El concreto es más pesado con las sustituciones residuales como se evidencia en la Tabla 4. donde los porcentajes del 20, 15 y $5 \%$ de lodo aluminoso presentaron pesos de $3.17,2.94$ y $2.72 \mathrm{~kg}$ respectivamente mucho mayores que 2.55 $\mathrm{kg}$ en la estructura de referencia; estas diferencias pueden ser atribuidas al tamaño de partícula y la humedad que influyen en la compactación de las mezclas.

Tabla 5: Características del concreto en relación al porcentaje de adición

\begin{tabular}{lccccc}
\hline \multirow{2}{*}{ Propiedades } & \multicolumn{5}{c}{ Porcentaje de adición (\%) } \\
\cline { 2 - 5 } & Referencia & 5 & 10 & 15 & 20 \\
\hline Materia orgánica (\%) & 0.15 & 0.19 & 0.25 & 0.39 & 0.52 \\
Densidad (g/mL) & 1.37 & 1.83 & 1.81 & 1.82 & 1.63 \\
Porosidad (\%) & 6.58 & 7.35 & 7.40 & 7.60 & 7.80 \\
Contracción lineal (\%) & 2.00 & 3.00 & 2.00 & 2.00 & 2.00 \\
Peso(kg) & 2.55 & 2.94 & 3.12 & 3.17 & 3.17 \\
Absorción de agua (\%) & 1.75 & 1.70 & 1.85 & 2.02 & 2.55 \\
\hline
\end{tabular}

Los esfuerzos máximos a los que se somete el concreto con lodos aluminosos justo antes de agrietarse o romperse en los ensayos mostraron una diferencia significativa ( $F=9.999 ; p=0.001 ; n=48)$, donde los valores más altos se presentaron con porcentajes del 15 y $20 \%$, se corresponden a materiales no estructurales de acuerdo con los límites del estándar ASTM-C129, y los más bajos 5 y 10\% no cumplen en su mayoría con los límites mínimos de las norma ASTM-C90 y ASTM- C129 para materiales de construcción estructural y no estructural respectivamente figura 3. Las razones de la disminución seguramente se deben a la textura de la mezcla, dando como resultados el desarrollo de grietas alrededor de las partículas durante la carga, lo que conduce una rápida falla de las muestras por la distribución no uniforme de los esfuerzos entre las partículas, en comparación con las mezclas de cemento y el agregado natural. Las variaciones en la absorción de agua en el concreto en relación a la incorporación de lodos aluminosos oscilaron entre 1.70 y $2.55 \%$ indicando una relación directamente proporcional, causando mayor porosidad.
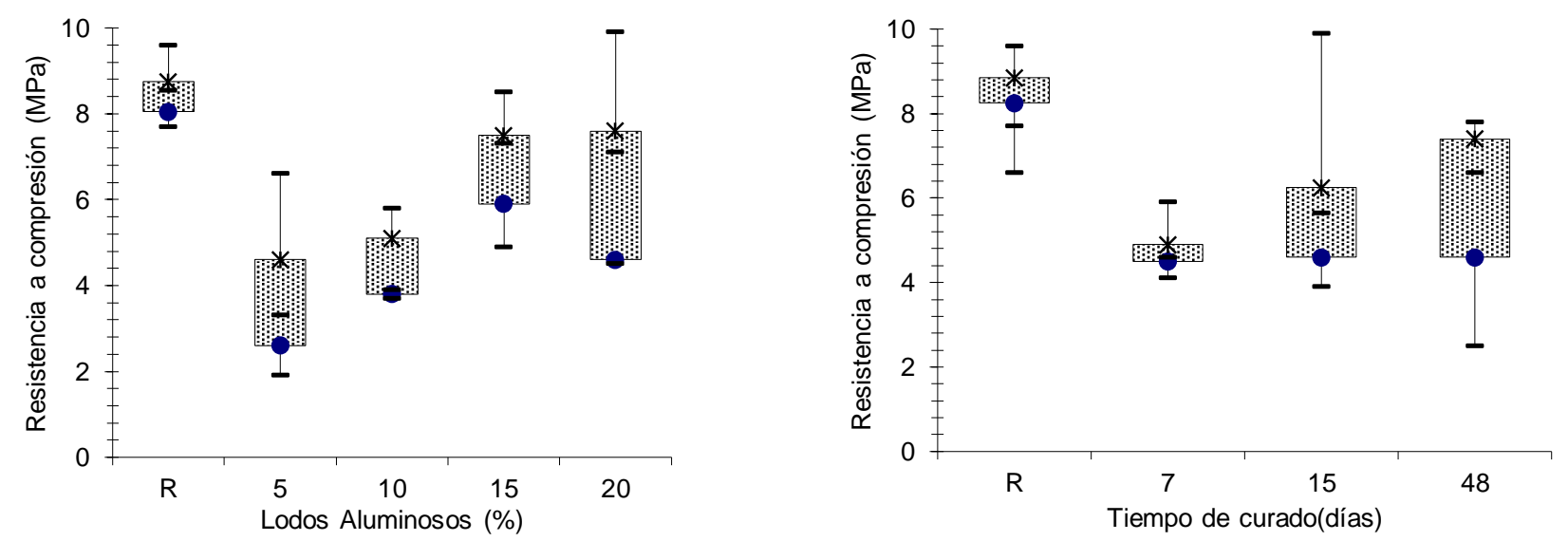

Fig. 3: Resistencia a la compresión del concreto con adiciones del 5, 10,15 y $20 \%$ de lodo aluminoso, a los 7, 28 y 48 días de curado en comparación con la referencia. 


\section{DISCUSIÓN}

Los altos niveles de óxido de silicio, óxido de aluminio; y óxido de hierro presentes en el lodo aluminoso analizado en la presente investigación, viabilizan su uso, recuperación y acondicionamiento como material de construcción; cuando la solución ácida rica en aluminio se reutiliza, proporciona beneficios similares a los productos comerciales (Durante et al., 2018; Ramirez et al., 2017). Es necesario avanzar en la factibilidad y los análisis económicos que relacionen los costos y beneficios del proceso, como se menciona en las investigaciones de Wang et al., (2018) y de Dahhou et al., (2018) quienes consideran importante verificar la conveniencia de usarlo, los ahorros desde el punto de vista de las materias primas, y finalmente si es necesario para hacer cambios en el proceso.

Es viable utilizar el concreto con un $15 \%$ de reemplazo de lodo aluminoso y concuerda con los reportados por Liu et al., (2020) y Bohórquez et al., (2020) quienes evidencian un aumento en la resistencia a la compresión hasta porcentajes de lodo aluminoso de (5 y 15\%) de la misma manera evidenciaron una disminución en la resistencia a la compresión para los porcentajes más alto. Los porcentajes de adición del $15 \%$ presentaron los valores máximos de resistencia a la compresión, superando los valores del concreto de referencia ( $100 \%$ de cemento); contrario a las demás adiciones donde tiende a disminuir.

Tabla 6: Resistencia a la compresión de adiciones residuales evaluadas en la elaboración de concretos no estructurales por diferentes autores.

\begin{tabular}{lll}
\hline $\begin{array}{l}\text { Porcentaje de Adición } \\
(\%)\end{array}$ & $\begin{array}{l}\text { Resistencia a la } \\
\text { comprensión }\left(\mathrm{kg} / \mathrm{cm}^{2}\right)\end{array}$ & \multicolumn{1}{c}{ Referencias } \\
\hline 5,10 & $0.48-51.40$ & (Fontes et al., 2016) \\
5,10 & $5.00-20.00$ & (De Carvalho et al., 2019) \\
10,30 & $16.20-28.42$ & (Bohórquez et al., 2020) \\
$20,30,40,70$ & $49.30-87.11$ & (Cremades et al.,2018) \\
$5,15,20$ & $4.10-6.43$ & (Fuentes et al.,2019) \\
$20,30,40,50$ & $19.90-22.60$ & (Danish et al.,2020) \\
$5,10,15,20$ & $4.13-48.69$ & (Liu et al.,2020) \\
$10,20,30$ & $0.15-0.31$ & (Rebolledo y Restrepo 2019) \\
$5,10,15,20$ & $83.00-248.30$ & (Rabie 2016) \\
$5,10,20$ & $35.20-76.40$ & (Ahmad et al., 2018) \\
\hline
\end{tabular}

En la Tabla 6 se evidencia los porcentajes del 10\% cumplen con la norma para su uso como material de construcción no estructural y estructural ASTM-C129 y ASTM-C90; según las investigaciones realizadas por Fontes et al., (2016), Danish et al., (2020), y Rebolledo y Restrepo (2019), se recomienda tener en cuenta este porcentaje en futuras investigaciones para revisar si es posible obtener mejores resultados en la resistencia a la compresión y maximizar las demás propiedades.

El contenido de materia orgánica del concreto elaborado con lodos aluminosos es ligeramente mayor que la referencia, se esperaría que este contenido pueda ser reducido al aplicar un tratamiento previo que mejore los resultados, como los reportes de Durante et al., (2018) y Fuentes et al., (2019) quienes calcinaron los lodos para maximizar las propiedades del concreto y reducir los procesos de oxidación de la materia orgánica en el tiempo. Adicionalmente, el concreto con sustitución de lodos aluminosos, presentaron mayor grado de contracción, mayor porosidad y, por ende, mayor capacidad para absorber agua, lo cual permite inferir que estos materiales presentarán una tendencia al desgaste y a la hidratación, por lo cual se sugiere ubicarlos en lugares preferenciales en comparación con los comerciales elaborados de solo cemento.

La Tabla 7 muestra un ahorro hasta del $40 \%$ en el costo de elaboración del concreto con adiciones de lodos aluminosos con sustitución parcial del cemento en la elaboración de 1000 cilindros, el cual es un ahorro directo del requerimiento de cemento, siendo este uno de los productos del sector industrial que aporta cerca del 7 $\%$ de la producción de $\mathrm{CO}_{2}$ causante del efecto invernadero a nivel mundial (Rabie, 2016); dando paso a los beneficios ambientales al reducir la contaminación por las cantidades de lodos aluminosos vertidos al ambiente, sin embargo es necesario realizar estudios de factibilidad económica, que permitan ampliar y soportar las acciones de manejo y disposición final de estos lodos. La investigación analiza los costos, evidenciando la rentabilidad y viabilidad en los aspectos ambientales, económicos y sociales. 
Tabla 7: Relación del coste del concreto con adiciones de lodos aluminosos en relación con la mezcla de referencia.

\begin{tabular}{lcc}
\hline $\begin{array}{l}\text { Porcentaje de } \\
\text { adición (\%) }\end{array}$ & $\begin{array}{c}\text { Resistencia } \\
(\mathrm{MPa})\end{array}$ & $\begin{array}{c}\text { Costo }(€) / 1000 \\
\text { cilindros }\end{array}$ \\
\hline $5 \%$ & 4.17 & 54.76 \\
$10 \%$ & 6.17 & 47.53 \\
$15 \%$ & 6.47 & 42.59 \\
$20 \%$ & 6.73 & 36.51 \\
Referencia & 8.65 & 60.84 \\
\hline
\end{tabular}

Los investigadores Ahmad et al. (2018) y Fuentes et al. (2019) argumentan que la reutilización de lodos como material de construcción plantea una interesante línea de investigación no desarrollada que, si se materializa, representa una solución para la eliminación de los mismos y una disminución del impacto ambiental en los canales de agua debido a la reducción de las cantidades vertidas en el medio ambiente. De lo anterior se puede especificar que en el campo de la ingeniería puede ser interesante desarrollar nuevos materiales de construcción, a partir de residuos que constituyen un problema ambiental. Y también con el uso de lodo, es posible reducir las cantidades de este material descargadas en el medio ambiente, reduciendo los niveles de contaminación y produciendo un beneficio económico, generando un material de construcción útil para ciertos usos cuyo costo económico es menor con los materiales convencionales.

\section{CONCLUSIONES}

De acuerdo a los resultados obtenidos y análisis presentados, se pueden extraer las siguientes conclusiones principales:

1.- Los lodos aluminosos son un agregado útil como material de construcción, es una fuente de hierro y aluminio que les confiere mayor resistencia a la compresión, sin modificar la apariencia física de la textura, forma y consistencia visual del concreto:

2.- Los mejores reemplazos son del 15 y $20 \%$ de lodo aluminoso, clasifican como materiales de construcción no estructurales, cumplen con los límites mínimos de $6.8 \mathrm{MPa}$ establecidos norma INVE-410 y son similares a los disponibles comercialmente; y

3.- Es una alternativa ambiental innovadora, que nos permite avanzar con un instrumento económicamente viable y socialmente aceptado, para potenciar las buenas prácticas de la industria de la construcción, logrando promover una economía circular que convierte los lodos residuales en un nuevo material.

\section{REFERENCIAS}

Ahmad, T., Ahmad, K., y Alam, M., Investigating calcined filter backwash solids as supplementary cementitious material for recycling in construction practices, https://doi.org/10.1016/j.conbuildmat.2018.04.227, Constr. Build. Mater., 175, 664$671(2018)$

Bohórquez, K., Pacheco, E., y otros 4 autores, Use of sludge ash from drinking water treatment plant in hydraulic mortars, https://doi.org/10.1016/j.mtcomm.2020.100930, Materials Today Communications, 23, 1-10 (2020)

Chen, Q., An, X., Li, H., y otros 3 autores., Long-term field application of sewage sludge increases the abundance of antibiotic resistance genes in soil, https://doi.org/10.1016/j.envint.2016.03.026, Environment International, 92, 1-10 (2016)

Cremades, L., Cusidó, J., y Arteaga, F., Recycling of sludge from drinking water treatment as ceramic material for the manufacture of tiles, https://doi.org/10.1016/j.jclepro.2018.08.094, J. Clean. Prod., 201, 1071-1080 (2018)

Danish, P., Ganesh, S., Raina, C., Implementation of paper mill waste as partial replacement material, https://doi.org/10.17485/IJST/v13i19.583, Indian Journal of Science and Technology, 13(19),1901-1907 (2020)

Dahhou, M., El Moussaouiti, M., y otros 3 autores, Synthesis and characterization of drinking water treatment plant sludge-incorporated Portland cement, https://doi.org/10.1007/s10163-017-0650-0, J. Mater. Cycles. Waste. Manag., 20, 891-901 (2018)

Dassanayake, K.B., Jayasinghe, G.Y., Surapaneni, A., y Hetherington, C., A review on alum sludge reuse with special reference to agricultural applications and future challenges, http://doi.org/10.1016/j.wasman.2014.11.025, Waste Management, 38, 321-335 (2015)

De Carvalho, S., Zhou, J.L., Li, W., y Long, G., Progress in manufacture and properties of construction materials incorporating water treatment sludge: a review, https://doi.org/10.1016/j.resconrec.2019.02.032, Resources Conserve \& Recycling, 145, 148-159 (2019) 
Durante, M., Camarini, G., y Murilo, F., Performance of mortars with the addition of septic tank sludge ash, https://doi.org/10.1016/j.conbuildmat.2017.11.053, Constr. Build. Mater., 160, 308-315 (2018)

Fontes, C.M., Toledo, R.D., y Barbosa, M.C, Cinza de lodo de esgoto (CLE) em concretos de alto desempenho: caracterização e aplicação, http://dx.doi.org/10.1590/S1983-41952016000600009, Rev. IBRACON Estrut. Mater., 9(6), 989-1006 (2016)

Franco, J., Varela, T., y otros 4 autores, More eco-efficient concrete: an approach on optimization in the production and use of waste-based supplementary cementing materials, https://doi.org/10.1016/j.conbuildmat.2019.02.054, Constr. Build. Mater., 206(1), 397- 409 (2019)

Fuentes, N., Isenia, S., y Ascencio, J., Adicion de lodos residuales en la elaboracion de matrices de cerámicas, https://doi.org/10.24050/reia.v16i32.1061, Rev. EIA. Esc. Ing. Antioq., 16(32), 13-25 (2019)

Gastaldini, A.L., Hengen, M.S., y otros 4 autores, The use of water treatment plant sludge ash as a mineral addition, https://doi.org/10.1016/j.conbuildmat.2015.07.038, Constr. Build. Mater., 94, 513-520 (2015)

Gessa, A., Sancha, M., Alternativas de reducción de las emisiones de dióxido de carbono (CO2) en la producción de cemento, Propuesta de un modelo de evaluación, http://dx.doi.org/10.15446/innovar.v26n60.55532, Innovar, 26(60), 51$66(2016)$

Hagemann, S.E., Gastaldini, A.L., y otros 3 autores, Synergic effects of the substitution of Portland cement for water treatment plant sludge ash and ground limestone: technical and economic evaluation, https://doi.org/10.1016/j.jclepro.2018.12.324, J. Clean. Prod., 214(1), 916-926 (2019)

Ibrahim, M., Hamzah, A., Jamaluddin, N., Mangi, S., y otro autor, Influence of bottom ash as a sand replacement material on durability of self-compacting concrete exposed to seawater, http:

//jestec.taylors.edu.my/Vol\%2015\%20issue\%201\%, .Journal of Engineering Science and Technology (JESTEC), 15(1), 555-571 (2020).

Kluczka, J., Zołotajkin, M., Ciba, J., y otro autor, Evaluación de la biodisponibilidad del aluminio en lodos de alumbre para uso agrícola, http://dx.doi.org/10.1007/s10661-017-6133-x, Environt Monit Assessment, (8) 189,422, (2017)

León, A., y Guillén, V., Energía contenida y emisiones de CO2 en el proceso de fabricación del cemento en Ecuador. https://doi.org/10.1590/s1678-86212020000300448, Ambiente Construido, 20 (3), 611-625 (2020)

Liu, Y., Zhuge, Y., y otros 6 autores, Utilization of drinking water treatment sludge in concrete paving blocks: microstructural analysis, durability and leaching properties, https://doi.org/10.1016/j.jenvman.2020.110352, J. Environ. Manage., 262(20), 1-9 (2020)

Masłon, A., Czarnota, J., Szaja A., y otros 2 autores., The enhancement of energy efficiency in a wastewater treatment plant through sustainable biogas use: case study from Poland, https://doi.org/10.3390/en13226056, Energies, (13), 1-21 (2020)

Medeiros, L., Durante, L., Callejas, I., Contribuição para a avaliação de ciclo de vida na quantificação de impactos ambientais de sistemas construtivos. Ambiente Construído, https://doi.org/10.1590/s1678-86212018000200259, porto Alegre, 2(18), 365-385 (2018)

Odimegwu, T.C., Zakaria, I., Abood, M.M., y otros 2 autores, Review on different beneficial ways of applying alum sludge in a sustainable disposal manner, http://dx.doi.org/10.28991/cej-03091153, Civil Engineering Journal, (4), 9,22302241 (2018)

Rabie, G.M., Using of wastewater dry and wet sludge in concrete mix, https://doi.org/10.4172/2165-784X.1000209, J. Civil. Environ. Eng., 6(1), 2-7 (2016)

Ramirez, K.G., Possan, E., Dezen, B.G., y Colombo, M., Potential uses of waste sludge in concrete production, https://doi.org/10.1108/MEQ-09-2015-0178, Manag. Environ. Qual., 28(6), 821-838 (2017)

Rebolledo, G., y Restrepo, I., Potencial de aprovechamiento de lodos industriales en la fabricación de pegante cerámico gris para pisos, https://doi.org/10.25100/iyc.v22i1.8023, InyCompe., 22(1), 1-12 (2019)

Smol, M., Kulczycka J., y otros 3 autores, The possible use of sewage sludge ash (SSA) in the construction industry as a way towards a circular economy, https://doi.org/10.1016/j.jclepro.2015.02.051, J. Clean. Prod., 95, 45-54 (2015)

Vatachi, N., Wastewater sludge to energy production, A review, https://doi.org/10.1088/1757-899X/595/1/012053, IOP Conf. Ser. Mater. Sci. Eng., 595 (2019)

Vouk, D., Nakic, D., Stirmer, N., y Cheeseman C., Influence of combustion temperature on the performance of sewage sludge ash as a supplementary cementitious material, https://doi.org/10.1007/s10163-018-0707-8, J. Mater. Cycles. Waste. Manag. 20, $1458-1467$ (2018)

Wang, L., Zou, F., y otros 5 autores, A novel type of controlled low strength material derived from alum sludge and green materials, https://doi.org/10.1016/j.conbuildmat.2018.01.078, Constr. Build. Mater. 165, 792-800 (2018)

Zhang, J., Cheng, J., y Lo, I., Life cycle carbon footprint measurement of Portland cement and ready mix concrete for a city with local scarcity of resources like Hong Kong, Int J Life Cycle Assess, https://doi.org/10.1007/s11367-013-0689-7, (19), 4, 745-757 (2014) 